\author{
А.Е. Шкляев, А.С. Пантюхина*, А.А. Сапегин, Е.Л. Баженов \\ ФГБОУ ВО «Ижевская государственная медицинская академия» Минздрава России, \\ кафедра факультетской терапии с курсами эндокринологии и гематологии, Ижевск, Россия
}

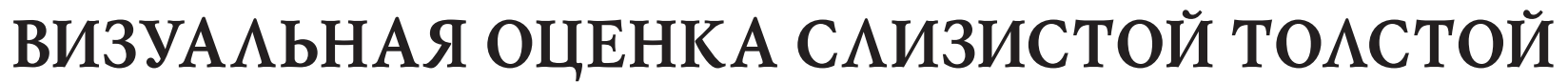 КИШКИ ПРИ СИНАРОМЕ РАЗАРАЖЕННОГО КИШЕЧНИКА В АИНАМИКЕ АЕЧЕНИЯ
}

\author{
A.E. Shklyaev, A.S. Pantyukhina*, A.A. Sapegin, E.L. Bazhenov \\ Izhevsk State Medical Academy Russian Ministry of Health, Department of faculty therapy \\ with courses in endocrinology and hematology, Izhevsk, Russia

\section{VISUAL ASSESSMENT OF THE MUCOSA OF THE COLON IN IRRITABLE BOWEL SYNDROME IN THE DYNAMICS OF TREATMENT}

\begin{abstract}
Резюме
Введение. Синдром раздраженного кишечника (СРК) - распространенное функциональное заболевание желудочно-кишечного тракта. Трудности его диагностики связаны с отсутствием специфических маркеров заболевания. В статье описаны визуальные изменения слизистой толстой кишки при синдроме раздраженного кишечника в динамике медикаментозного и физиотерапевтического лечения. Материалы и методы. Обследовано и пролечено 100 больных с эндоскопически подтвержденным диагнозом СРК. Из которых 50 человек получали фармакотерапию и 50 - СМТ - форез природного рассола. Дана морфологическая оценка слизистой кишечника в динамике лечения. Результаты. При СРК морфо-эндоскопически выявляются дискинетические нарушения, признаки неспецифического минимального воспаления слизистой толстой кишки и начальные дистрофические проявления. Данные изменения носят обратимый характер и могут использоваться для оценки качества лечения. Установлена высокая эффективность СМТ-фореза природного рассола при СРК.
\end{abstract}

Ключевые слова: колоноскопия, синдром раздраженного кишечника, СМТ-форез.

\begin{abstract}
Introduction. Irritable bowel syndrome (IBS) is a common functional disorder of the gastrointestinal tract. The difficulty of its diagnosis related to the lack of specific markers of the disease. This article describes the visual changes of the mucosa of the colon in irritable bowel syndrome in the dynamics of medical and physiotherapy treatment. Materials and methods. Examined and treated 100 patients with endoscopically confirmed diagnosis of IBS. Of which 50 people were receiving pharmacotherapy and 50 -sinusoidal modulated currents - phoresis natural brine. Given the morphological evaluation of the mucosa of the intestine in the dynamics of treatment. Results. In IBS morpho-endoscopically detected dyskinetic disorders, minimal signs of nonspecific inflammation of the mucosa of the colon and primary degenerative manifestations. These changes are reversible and can be used to assess the quality of treatment. The high efficiency of SMC-phoresis natural brine in IBS.
\end{abstract}

Key words: colonoscopy, irritable bowel syndrome, SMC-phoresis.

DOI: $10.20514 / 2226-6704-2016-6-6-34-37$

СРК - синдром раздраженного кишечника

\section{Введение}

Синдром раздраженного кишечника (СРК) является функциональным расстройством и диагностируется на основании симптомов заболевания, изложенных в Римских критериях IV (2016г), с исключением органической патологии. С дифференциально-диагностической целью проводится колоноскопия с ос- мотром терминального отдела подвздошной кишки и прицельной биопсией. С ее помощью можно исключить воспалительные заболевания кишечника, микроскопические колиты, новообразования, нейроэндокринные опухоли, дивертикулы толстой кишки и другие заболевания [3, 4, 5]. По литературным данным при СРК эндоскопически визуализируются нарушения моторно-эвакуаторной деятельности

*Контакты/Contacts. E-mail: pantyukhina1985@yandex.ru 
кишечника, а также минимальные признаки неспецифического воспаления толстой кишки [1, 3, 4]. Поэтому возможно проведение колоноскопии в динамике для контроля лечения СРК, однако работы на эту тему представлены в единичных случаях и касаются исследования действия конкретных препаратов. Стандартная фармакотерапия направлена на устранение спазмов, нормализацию стула и психоэмоционального фона больного и включает в себя комплексное назначение препаратов, в том числе психотропных. Однако современное медикаментозное лечение в большинстве случаев бывает малоэффективным и может вызывать побочные реакции [3]. В связи с этим продолжается поиск новых методов лечения, которые бы одновременно воздействовали на ведущие звенья патогенеза, повышали эффективность терапии и способствовали ограничению применения медикаментозных препаратов у пациентов с СРК. Перспективным для восстановления функциональной активности организма является использование природных факторов, с подтверждением лечебного действия на ультраструктурном уровне.

Цель исследования: эндоскопическая и морфологическая оценка слизистой кишечника при СРК в динамике медикаментозного и физиотерапевтического лечения.

\section{Материалы и методы}

Обследовано и пролечено 100 пациентов с различными формами СРК. Распределение по группам осуществлялась методом случайной выборки. Аиагноз верифицирован на основании «Римских критериев III» (2006) и подтвержден эндоскопически. Пациенты были разделены на две равные группы. В группе наблюдения проводился СМТ-форез хлоридного бромйодсодержащего рассола санатория «Варзи-Ятчи» (Удмуртская Республика) по следующей методике: анод от аппарата (Амплипульс-5) накладывался вместе с прокладкой смоченной рассолом температурой $38^{\circ} \mathrm{C}$ на мезогастральную область. Катод на область L1-L2. Прокладки с рассолом были оставлены на коже ещё 10-30 минут после проведения сеанса СМТ-фореза в зависимости от переносимости процедуры. При диарейном синдроме СМТ проводилось по щадящей методике (III и IV роды работ, по 5 мин. каждый, частота 100 Гц, глубина модуляций 50\%), при запорах - по стимулирующей методике (III и II роды работ, по 5 мин. каждый, частота 50 70 Гц, глубина модуляций 75-100\%). Курс состоял из 10-12 процедур. Пациенты группы сравнения получали стандартную фармакотерапию при СРК (мебеверина гидрохлорид, лактулозу - при запоре, амитриптилин по показаниям) $[3,4]$.

Перед началом терапии и по ее окончанию проводилась эндоскопическая оценка состояния слизистой оболочки кишечника с помощью видеоколоноскопии (82\%) и ректороманоскопии (18\%). При исследовании уделялось внимание состоянию слизистой кишечника и дисмоторным нарушениям, диагностированным по расстоянию между складкми, их высоте и ширине. Колоноскопия проводилась на аппарате фирмы “Олимпус» с осмотром терминального отдела подвздошной кишки и прицельной биопсией по 1-2 кусочка с зон толстой кишки, где имелись изменения слизистой. Биоптаты подвергались гистологическому и электроно-микроскопическому исследованию.

Исследование одобрено Комитетом по биоэтике ФГБОУ ВО (ИГМА» МЗ РФ. Аечение проводилось на основе информированного согласия больного.

Анализ результатов исследования осуществлен с помощью стандартных компьютерных программ for Window, Microsoft Office Excel, 2007; Statistica v. 6,0.

\section{Результаты исследования}

Группы были сопоставимы по основным характеристикам (табл. 1). Ао начала курсовой терапии эндоскопически не выявлено значительных визуальных изменений слизистой оболочки кишечника, что соответствует современному представлению о СРК как функциональной патологии. Результаты проводимой в динамике эндоскопии представлены в таблице 2.

у большинства пациентов группы наблюдения $(62,0 \%)$ и сравнения (64,0\%) исходная картина слизистой оболочки тонкой и толстой кишок соответствовала норме - она имела розовую окраску, была блестящей, гладкой, с хорошо видимым сосудистым рисунком, с небольшим наложением слизи (рис 1).

Нарушения моторики толстой кишки выявлялись у 52\% всех обследованных (рис. 2).

Смазанный сосудистый рисунок, очаговая гиперемия слизистой (рис. 3) и отечность складок кишечника в основном наблюдались у пациентов с диареей.

Таблица 1. Характеристика групп

Table 1. Dynamics of endoscopic picture in IBS patients

\begin{tabular}{|c|c|c|}
\hline & Группа & Группа \\
& наблюдения & сравнения \\
& $(50$ человек)/ & $(50$ человек)/ \\
& Monitoring & The control \\
& Group (n=50) & group $(\mathrm{n}=50)$ \\
\hline
\end{tabular}

Средний возраст/

Average age

$28,01 \pm 8,87$

$23,9 \pm 9,67$

Женщины: мужчины/

Women: Men

$1,7: 1$

$2,3: 1$

Запоры/Constipation (\%)

60

54

Аиарея/Diarrhea (\%)

40 
По окончанию курсового СМТ-фореза лечебного рассола наблюдалось увеличение доли пациентов с визуально неизмененной слизистой до 82,0\%, после медикаментозного лечения - до 78,0\%. СМТ-форез хлоридного бромйодсодержащего рассола оказал большее положительное влияние в отношении гиперсекреции слизи, отека и очаговой гиперемии слизистой кишечника в сравнении с медикаментозной терапией. Нарушения моторики кишечника в группе наблюдения после лечения встречались лишь в единичных случаях, в группе сравнения результаты были менее выраженными. Важно отметить лучшую переносимость пациентами колоноскопии после лечения. Частота субъективных болезненных ощущений при проведении данной процедуры снизилась с 24,0\% до 4,6\% - в группе наблюдения и до 12,8\% сравнения.

Морфологическое исследование биоптатов слизистой оболочки толстой кишки проведено у 74 паци- ентов, имевших эндоскопические отклонения от нормы. Полученные данные гистологических исследований слизистой кишечника показали, что у пациентов обеих групп до лечения в 44,4\% случаев имелись морфологические признаки минимальных воспалительных изменений в виде смешанного инфильтрата в собственной пластинке слизистой и полнокровия сосудов микроциркуляторного русла (рис. 4).

В 48,6\% случаев выявлялись признаки дистрофического процесса в виде вакуолизации цитоплазмы и набухания митохондрий. Столь высокий процент изменений, вероятно, связан с забором материала с визуально измененных участков слизистой подвздошной и толстой кишки. После курсового лечения минимальные признаки воспаления в группе наблюдения встречались в 29,3\% случаев, в группе сравнения - в 39,4\%. Аистрофические проявления встречались в группе наблюдения в 1,4 раза реже, чем в группе сравнения.

Таблица 2. Аинамика эндоскопических показапелей

Table 2. Dynamics of endoscopic picture in IBS patients

\begin{tabular}{|c|c|c|c|c|}
\hline \multirow[b]{2}{*}{ Эндоскопические показатели/ Endoscopic indicators } & \multicolumn{2}{|c|}{ Группа наблюдения, \% } & \multicolumn{2}{|c|}{$\begin{array}{c}\text { Группа сравнения } \\
(\mathrm{n}=\mathbf{5 0}), \%\end{array}$} \\
\hline & $\begin{array}{c}\text { До } \\
\text { лечения/ } \\
\text { Before } \\
\text { treatment }\end{array}$ & $\begin{array}{c}\text { После } \\
\text { лечения/ } \\
\text { After } \\
\text { treatment }\end{array}$ & $\begin{array}{c}\text { Ао } \\
\text { лечения/ } \\
\text { Вefore } \\
\text { treatment }\end{array}$ & $\begin{array}{c}\text { После } \\
\text { лечения/ } \\
\text { After } \\
\text { treatment }\end{array}$ \\
\hline Визуально неизмененная слизистая/ The visually unchanged mucosa & 62,0 & 82,0 & 64,0 & 78,0 \\
\hline Большое количество слизи/Large amounts of mucus & 40,0 & 12,0 & 38,0 & 18,0 \\
\hline Очаговая гиперемия слизистой/Focal hyperemia mucous & 16,0 & 4,0 & 20,0 & 6,0 \\
\hline Смазанный сосудистый рисунок/ Oiled vascular pattern & 14,0 & 4,0 & 12,0 & 6,0 \\
\hline Небольшая отечность слизистой/A small edema of of the mucous & 16,0 & 4,0 & 18,0 & 8,0 \\
\hline Сглаженность складок/Smoothness folds & 4,0 & 2,0 & 4,0 & 4,0 \\
\hline Гипертонус кишки/Gipertonus colon & 34,0 & 2,0 & 28,0 & 4,0 \\
\hline Гипотония кишки/Gipotonus colon & 22,0 & 2,0 & 20,0 & 10,0 \\
\hline
\end{tabular}

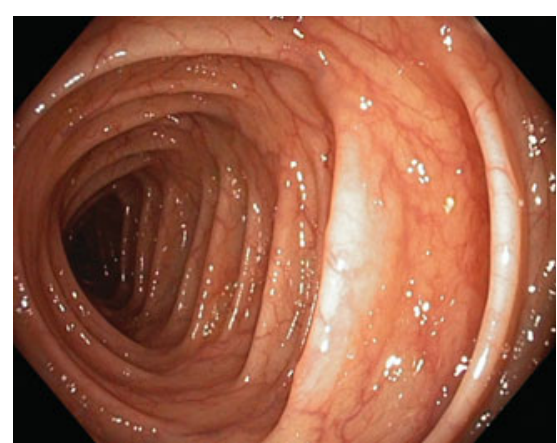

Рисунок 1. Слизистая толспой кишки с выраженным сосудиспым рисунком, без признаков воспаления, с небольшим наложением слизи, нормопонус.

Figure 1. The mucosa of the colon with marked vascular pattern, with no signs of inflammation, with little overlap mucus normotonus.

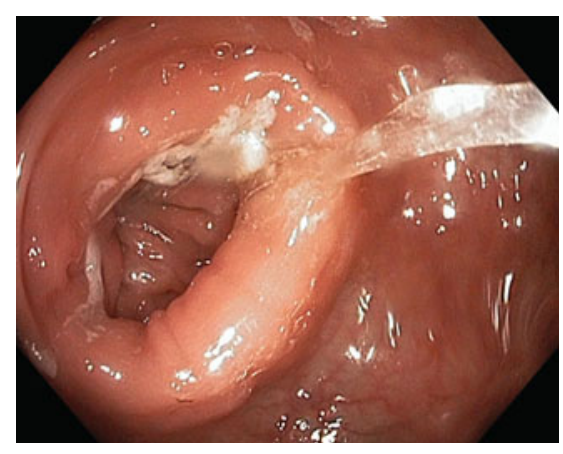

Рисунок 2. Гиперпонус полспой кишки с большим наложением слизи.

Figure 2. Hypertonicity of the colon with a large overlay mucus.

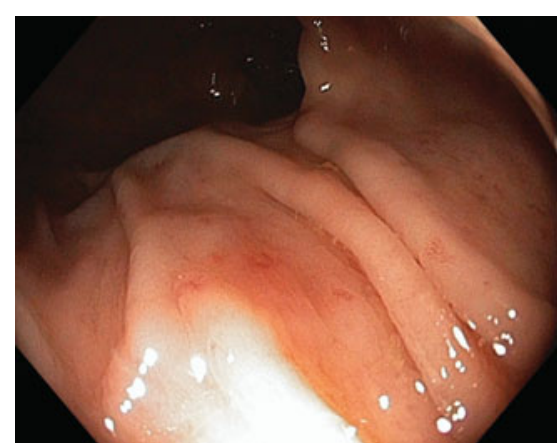

Рисунок 3. Смазанный сосудистый рисунок, очаговая гиперемия слизиспой.

Figure 3. Blurry vascular pattern, focal hyperemia of the mucous membrane. 


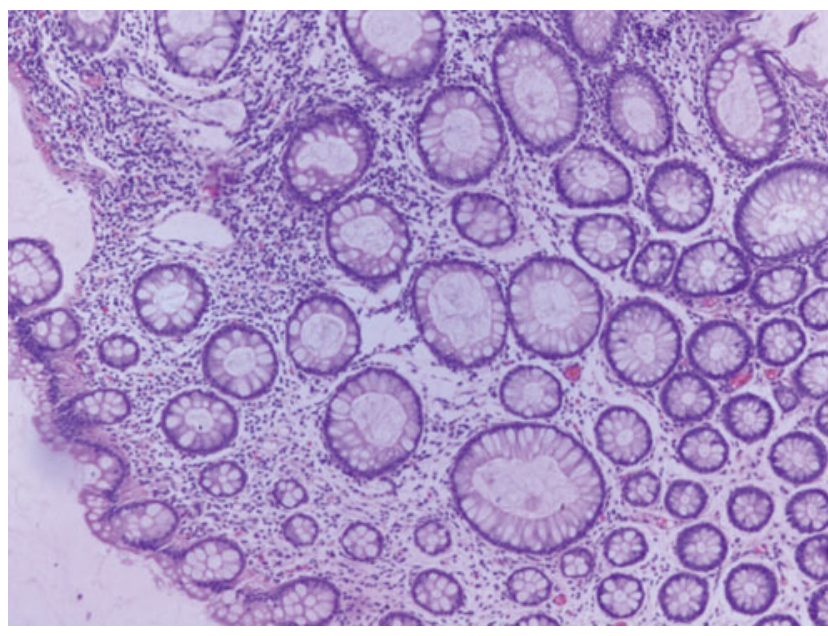

Рисунок 4. Смешанный инфильтрап, состоящий из лимфоцитов, плазмоцитов, макрофагов. Окраска гемапоксилином и эозином, $\times 280$.

Figure 4. A mixed infiltrate consisting of lymphocytes, plasma cells, macrophages. Hematoxylin and eosin, $\times 280$.

\section{Обсуждение}

В предполагаемом механизме возникновения минимального персистирующего воспаления при СРК играет роль нарушение проницаемости эпителиального слоя кишечной стенки, что приводит к увеличению количества проникающих бактериальных клеток, а затем к избыточному выбросу биологически активных веществ, таких как гистамин, лейкотриены, простагландины и фактор активации тромбоцитов, усиливающих проницаемость сосудов и поддерживающих воспаление на клеточном уровне [3].

Терапевтическое действие апробированного метода обусловлено параметрами электрического тока, влияющего на моторику толстой кики, и химическим составом рассола. Под влиянием СМТ происходит всасывание активных компонентов рассола, обеспечивающих лечебные эффекты. Хлорид натрия, определяющий тип лечебного рассола «ВарзиЯтчи), практически не всасывается под действием амплипульса, но оказывает мощное раздражающее действие на рецепторный аппарат кожи, что вызывает рефлекторную обратную связь, поступающую к ЦНС, и стимулирует гуморальный ответ с выбросом в кровь биологически активных веществ (таких как серотонин, интерлейкин-1). Таким образом, запускается каскадная реакция, обуславливающая трофикорегенераторный и противовоспалительные эффекты [2].

\section{Выводы}

1. Эндоскопические и морфологические исследования при СРК могут выявить дискинетические нарушения, признаки неспецифического минимального воспаления слизистой толстой кишки и начальные дистрофические проявления.

2. Выявленные изменения слизистой толстой кишки имеют обратимый характер.

3. СМТ-форез хлоридного бромйодсодержащего рассола способствует улучшению структурно-функциональной организации слизистой оболочки кишечника более выражено, чем стандартная фармакотерапия.

4. Визуальная оценка слизистой толстой кишки при СРК способствует выбору тактики лечения и может рекомендоваться для динамического наблюдения.

\section{Список литературы/References:}

1. Визе-Хрипунова М.А. Эндоскопические изменения толстой кишки у больных синдромом раздраженного кишечника Медицинский альманах. 2012; 20(1): 81-83.

Wiese-Khripunova M.A. Endoscopic changes in the colon in patients with irritable bowel syndrome. Medical Almanac. 2012; 20(1): 81-83 [in Russian].

2. Владимирский Е.В., Фильцагина Т.Н. История бальнеотерапии: механизмы и лечебные эффекты. Российский журнал восстановительной медицины. 2014; 2: 3-9

Vladimirskiy E.V., Filtsagina T.N. History balneotherapy: mechanisms and therapeutic effects. Russian Journal of regenerative medicine. 2014; 2: 3-9 [in Russian].

3. Ивашкин В.Т. и др. Клинические рекомендации Российской гастроэнтерологической ассоциации, Ассоциации колопроктологов России по диагностике и лечению больных с синдромом раздраженного кишечника. РЖГГК. 2014; 24(2): 92-101.

Ivashkin V.T. et al. Clinical recommendations of the Russian Gastroenterological Association, the Association of Russian Coloproctologists for the diagnosis and treatment of patients with irritable bowel syndrome. Russian journal Hepatology, Gastroenterology, Coloproctology. 2014; 24(2): 92-101 [in Russian].

4. Лоранская И.Д., Лаврентьева О.А. Синдром раздраженного кишечника: учебное пособие. М., 2011; 40 с

Loranskaya I.D., Lavrenteva O.A. Irritable bowel syndrome: a tutorial. M.: Fort print, 2011. 40 p. [in Russian].

5. Сорокина Е.А. и др. Роль инструментальных методов исследования (ирригоскопии и эндоскопии толстой кишки) в диагностике синдрома раздраженного кишечника. Сибирский журнал гастроэнтерологии и гепатологии. 2003; 16: 182-183. Sorokin E.A. et al. The role of imaging studies (barium enema and endoscopy of the colon) in the diagnosis of irritable bowel syndrome. Siberian Journal of Gastroenterology and Hepatology. 2003; 16: 182-183 [in Russian].

Авторы заявляют, чпо данная работа, её тема, предмеп и содержание не затрагивают конкурирующих интересов/ The authors state that this work, its theme, subject and content do not affect competing interests

Статья получена/article received 04.07.2016 г. 